\title{
Croissance en autotrophie et en mixotrophie de la microalgue Micractinium pusillum Fres. isolée d'un lagunage naturel : influence de la lumière et de la température Autotrophic and mixotrophic growth of microalgae Micractinium pusillum Fres. isolated from wastewater stabilization pond: influence of light and temperature
}

\author{
L. Bouarab, M. Loudiki et A. Dauta
}

Volume 15, numéro 1, 2002

URI : https://id.erudit.org/iderudit/705437ar

DOI : https://doi.org/10.7202/705437ar

Aller au sommaire du numéro

\section{Éditeur(s)}

Université du Québec - INRS-Eau, Terre et Environnement (INRS-ETE)

\section{ISSN}

0992-7158 (imprimé)

1718-8598 (numérique)

Découvrir la revue

Citer cet article

Bouarab, L., Loudiki, M. \& Dauta, A. (2002). Croissance en autotrophie et en mixotrophie de la microalgue Micractinium pusillum Fres. isolée d'un lagunage naturel : influence de la lumière et de la température. Revue des sciences de l'eau / Journal of Water Science, 15(1), 73-86. https://doi.org/10.7202/705437ar

\section{Résumé de l'article}

Dans le but d'étudier la dynamique des microalgues dans le lagunage naturel de Ouarzazate (sud du Maroc) et éventuellement sa modélisation, nous avons eu recours à la détermination des paramètres de croissance ( $\mu$ max, Iopt et b) selon le modèle de PEETERS et EILERS (1978). À cet effet, Micractinium pusillum, algue verte Chlorococcale se développant dans les bassins de lagunage de la station pilote de Ouarzazate, a été isolée et mise en culture sur milieu synthétique (DAUTA, 1982) enrichi en azote et en phosphore sous des conditions contrôlées de lumière et de température.

En autotrophie, l'application du modèle de PEETERS et EILERS (1978) à la croissance de Micractinium pusillum, a permis la détermination des paramètres : $\mu \max (0,58 \mathrm{j}-1)$, I opt $\left(182 \mu \mathrm{E} \cdot \mathrm{m}^{-\mathrm{Z}} \cdot \mathrm{s}^{-1}\right)$ et b $(0,1)$ à la température maximale de croissance de $35^{\circ} \mathrm{C}$. En mixotrophie et à la même température, ces paramètres sont respectivement de $0,85 \mathrm{j}-1,150 \mu \mathrm{E} \cdot \mathrm{m}^{-\mathrm{z}} \cdot \mathrm{s}^{-1}$ et 1 . Comparativement à la croissance en autotrophie, la croissance de Micractinium pusillum en mixotrophie, en présence d'acétate, a mis en évidence la variation, selon le régime trophique de l'algue, des paramètres de croissance $\mu$ max, Iopt et b. La croissance en mixotrophie, comportement probable dans le lagunage naturel, a conduit particulièrement à l'élévation du taux de croissance et du coefficient b. La variation du coefficient $b$ traduirait une certaine sténophotie de la microalgue en autotrophie et une tendance à l'euryphotie en mixotrophie. Cette étude a montré également que l'influence de l'intensité lumineuse sur la croissance varie en fonction du mode trophique. 


\title{
Croissance en autotrophie et en mixotrophie de la microalgue Micractinium pusillum Fres. isolée d'un lagunage naturel : influence de la lumière et de la température
}

\author{
Autotrophic and mixotrophic growth of microalgae \\ Micractinium pusillum Fres. isolated from \\ wastewater stabilization pond: influence of light \\ and temperature
}

L. BOUARAB ${ }^{*}{ }^{1}$, M. LOUDIKI ${ }^{1}$, A. DAUTA ${ }^{2}$

SUMMARY

In the wastewater stabilization pond, the growth of the microalgae depends on several factors such as temperature, light and nutriments. In addition to these factors, the presence of the organic matter has great influence on the development in such system. In the aim to study the dynamic of the microalgae in the wastewater stabilization pond of Ouarzazate city (south of Morocco) and possibly its modelling, the determination of the growth parameters ( $\mu$ max, Iopt and $\beta$ ) has been carried out according to the model of PEETERS and EILERS (1978). Indeed, Micractinium pusillum, a Chlorococcal green algae developing in the experimental wastewater stabilization pond of Ouarzazate city has been isolated and cultured under controlled conditions of light and temperature on synthetic medium (DAUTA, 1982) enriched in nitrogen and phosphorus.

In autotrophic conditions, application of the PEETERS and EILERS model to the growth of Micractinium pusillum, resulted in the determination of the parameters: $\mu \max \left(0,58 \mathrm{day}^{-1}\right)$, Iopt $\left(182 \mu \mathrm{E} \cdot \mathrm{m}^{-2} \cdot \mathrm{s}^{-1}\right)$ and $\beta(0,1)$ using the maximal growth temperature of $35^{\circ} \mathrm{C}$. In mixotrophic conditions under the same temperature, these parameters were respectively $0,85 \mathrm{day}^{-1}, 150 \mu \mathrm{E} \cdot \mathrm{m}^{-2} \cdot \mathrm{s}^{-1}$ and 1 .

According to the trophic mode, the growth of Micractinium pusillum revealed a variation of the parameters $\mu$ max, Iopt and $\beta$. The mixotrophic growth, probable behaviour in the wastewater stabilization pond, has induced particularly the elevation of the growth rate and the coefficient $\beta$. Also, this study

1. Laboratoire d'algologie, département de biologie, Faculté des sciences Semlalia, Université Cadi Ayyad, BP 2390 Marrakech, Maroc.

2. CESAC, UMR C 5576 CNRS-Université Paul Sabatier, 118 route de Narbonne, F-31062 Toulouse cedex 04 France.

* Correspondance. E-mail : Bouarab@ucam.ac.ma

Les commentaires seront reçus jusqu'au 31 mars 2003. 
showed that light intensity has varied effects on the growth of microalgae in the two trophic modes.

Key-words: Micractinium pusillum, growth, autotrophic, mixotrophic, light, temperature.

\section{RÉSUMÉ}

Dans le but d'étudier la dynamique des microalgues dans le lagunage naturel de Ouarzazate (sud du Maroc) et éventuellement sa modélisation, nous avons eu recours à la détermination des paramètres de croissance ( $\mu$ max, lopt et $\beta$ ) selon le modèle de PEETERS et EILERS (1978). À cet effet, Micractinium pusillum, algue verte Chlorococcale se développant dans les bassins de lagunage de la station pilote de Ouarzazate, a été isolée et mise en culture sur milieu synthétique (DAUTA, 1982) enrichi en azote et en phosphore sous des conditions contrôlées de lumière et de température.

En autotrophie, l'application du modèle de PEETERS et EILERS (1978) à la croissance de Micractinium pusillum, a permis la détermination des paramètres : $\mu \max \left(0,58 \mathrm{j}^{-1}\right), \mathrm{I}$ opt $\left(182 \mu \mathrm{E} \cdot \mathrm{m}^{-2} \cdot \mathrm{s}^{-1}\right)$ et $\beta(0,1)$ à la température maximale de croissance de $35^{\circ} \mathrm{C}$. En mixotrophie et à la même température, ces paramètres sont respectivement de $0,85 \mathrm{j}^{-1}, 150 \mu \mathrm{E} \cdot \mathrm{m}^{-2} \cdot \mathrm{s}^{-1}$ et 1 . Comparativement à la croissance en autotrophie, la croissance de Micractinium pusillum en mixotrophie, en présence d'acétate, a mis en évidence la variation, selon le régime trophique de l'algue, des paramètres de croissance $\mu$ max, Iopt et $\beta$. La croissance en mixotrophie, comportement probable dans le lagunage naturel, a conduit particulièrement à l'élévation du taux de croissance et du coefficient $\beta$. La variation du coefficient $\beta$ traduirait une certaine sténophotie de la microalgue en autotrophie et une tendance à l'euryphotie en mixotrophie. Cette étude a montré également que l'influence de l'intensité lumineuse sur la croissance varie en fonction du mode trophique.

Mots clés : Micractinium pusillum, croissance, autotrophie, mixotrophie, lumière, température.

\section{1 - INTRODUCTION}

Plusieurs espèces de microalgues semblent posséder des potentialités d'élimination des nutriments azotés et phosphorés contenus dans les eaux usées et, ainsi, sont utilisées dans les stations de traitement des eaux usées, tels que le lagunage naturel et le lagunage à haut rendement.

En milieu naturel, la distribution et l'abondance des microalgues sont déterminées par leur capacité physiologique à s'adapter à l'environnement physique et chimique (HEE- MOCK et RHEE, 1991). La succession des espèces phytoplanctoniques est souvent expliquée par la différence au niveau de leurs constantes écophysiologiques spécifiques (GUERRI et al., 1981). La croissance instantanée dépend, quant à elle, des variables externes : intensité lumineuse, photopériode, température, $\mathrm{pH}$, nutriments (rapport N/P et quotas cellulaires en $\mathrm{C}, \mathrm{N}, \mathrm{P}$ ), matière organique pour certaines espèces, concentrations en $\mathrm{CO}_{2}$ et en $\mathrm{O}_{2}$ et état physiologique des cellules (CAPERON et MEYER, 1972 ; NEILSON et LEWIN, 1974 ; SOEDER et STENGER, 1974 ; SENFT, 1978 ; RHEE et GOTHAM, 1980 ; 1981a, b ; HERZIG et FALKOWSKI, 1989 ; SAKSHAUG et al., 1989 ; GAO et 
al., 1993 ; LALIBERTÉ et DE LA NOUE, 1993 ; SHAFIK et al., 1997a, b). RHEE et GOTHAM (1981a, b) suggèrent que l'effet combiné de ces facteurs est plus important que celui de chaque facteur pris séparément.

Si l'action d'un facteur physique comme la turbulence est écartée (GROBBELAAR, 1994), en présence de teneurs en nutriments non limitantes, l'efficacité des systèmes utilisant les microalgues reste principalement contrôlée par le taux de pénétration de la lumière et les conditions thermiques (DE LA NOÜE et DE PAUW, 1988). L'influence de ces facteurs (énergie lumineuse disponible et température) a largement été décrite et modélisée par de nombreux auteurs (JONES, 1977 ; HARRIS, 1978 ; DAUTA, 1982 ; REYNOLDS, 1984). La température a une influence directe sur le métabolisme (vitesse d'assimilation, respiration, photosynthèse) et, par conséquent, sur le taux de croissance du phytoplancton (GOLDMAN, 1977). La lumière agit par son intensité et par la qualité du spectre (WYNEE et RHEE, 1986). La croissance des algues est limitée par la lumière si la moyenne de l'intensité lumineuse reçue est inférieure à ce qu'exige la saturation de la photosynthèse (KNOWLTON et JONES, 1996).

Dans les écosystèmes aquatiques, les premières tentatives de modélisation visant à simuler la dynamique d'une population algale, avaient tendance à considérer le phytoplancton comme une unité homogène (GUERRI et al., 1981). Les études menées par plusieurs auteurs (GUERRI et al., 1981 ; BRUNEL et al., 1982 ; DAUTA, 1982 ; BOUMNICH et al., 1990 ; DAUTA et al., 1990 ; TALBOT et al., 1991 ; BELKOURA et DAUTA, 1992 ; DERRAZ et al., 1995 ; SHAFIK et al., 1997a, b) font ressortir l'existence de réelles différences entre les espèces, et l'importance des constantes spécifiques, en tant que paramètres explicatifs de la succession des espèces. L'étude de la dynamique ainsi que la valorisation des microalgues restent liées à la connaissance de leurs caractéristiques biochimiques et écophysiologiques. La détermination in situ de ces caractéristiques étant très difficile, nous nous sommes orientés vers une culture en laboratoire sur milieu synthétique. L'objectif de ce travail était d'étudier l'effet de la lumière et de la température sur les caractéristiques de croissance de Micractinium pusillum Fres. en conditions d'autotrophie et de mixotrophie en présence d'acétate, produit abondant dans les eaux usées.

\section{2 - MATÉRIEL ET MÉTHODES}

L'espèce Micractinium pusillum Fres., a été isolée à partir du lagunage naturel pilote de Ouarzazate (sud du Maroc). La culture en milieu fini (batch) est réalisée dans le milieu minéral proposé par DAUTA (1982). Dans le cas de la culture en mixotrophie, ce milieu est enrichi avec de l'acétate de sodium. La culture mère de $M$. pusillum est maintenue en phase exponentielle de croissance par des repiquages fréquents dans un milieu enrichi en nutriments, sous des conditions suboptimales de température $\left(25^{\circ} \mathrm{C}\right)$ et de lumière $\left(200 \mu \mathrm{E} \cdot \mathrm{m}^{-2} \cdot \mathrm{s}^{-1}\right)$ et une photopériode de 15 heures de lumière pour 9 heures d'obscurité. L'éclairement est dispensé par des lampes PhytoClaude de 400 watts placées à la verticale des cultures. La mesure des intensités lumineuses a été réalisée à l'aide d'un quantamètre Li - Cor Model Li - 189. 
L'expérience destinée aux mesures de la croissance en fonction de la température et de la lumière est réalisée selon le protocole décrit par DAUTA (1982). Pour chaque température $\left(15^{\circ}, 20^{\circ}, 25^{\circ}, 30^{\circ}, 35^{\circ}, 40^{\circ} \mathrm{C}\right)$, les cultures sont soumises à des intensités lumineuses comprises entre 8 et $630 \mu \mathrm{E} \cdot \mathrm{m}^{-2} \cdot \mathrm{s}^{-1}(8,20$, $\left.30,50,60,80,100,130,180,320,450,630 \mu \mathrm{E} \cdot \mathrm{m}^{-2} \cdot \mathrm{s}^{-1}\right)$, avec un éclairage vertical.

L'effet de l'acétate sur la croissance est étudié sous les mêmes conditions de lumière aux températures $20^{\circ}$ et $35^{\circ} \mathrm{C}$ sur milieu enrichi, d'après ENDO et al. (1977), avec de l'acétate à raison de $10 \mathrm{mM}$ (acétate de sodium).

\subsection{Estimation du taux de croissance}

L'équation permettant de décrire l'évolution d'une population $A$ en fonction du temps t s'écrit :

$$
\mathrm{dA} d \mathrm{dt}=\mu \mathrm{A}
$$

Le taux de croissance $(\mu)$ de la population à chaque instant peut être considéré comme le taux maximal spécifique de croissance ( $\mu$ max), affecté de facteurs de réduction liés aux variables : Iumière (Red I), température (Red $T)$, et aux éléments nutritifs : Azote (Red N) et Phosphore (Red P).

$$
\mu=\mu \max * \frac{\operatorname{Red} I^{*} \operatorname{Red} T}{\text { terme } 1}{ }^{*} \frac{\operatorname{Red} N{ }^{*} \operatorname{Red} \mathrm{P}}{\text { terme } 2}
$$

où le symbole « * " représente une combinaison des différents facteurs.

Pour étudier l'influence de la lumière et de la température (i.e. terme 1 de l'équation 2) sur la croissance, les nutriments sont apportés en excès ; le terme 2 de l'équation 2 est ainsi annulé (GUERRI et al., 1981).

Dans les conditions optimales de croissance, le taux de croissance $\mu$ est calculé à partir de deux évaluations quantitatives d'une population d'algues, réalisées respectivement aux temps $t_{1}$ et $t_{2}$, selon l'équation :

$$
\mu\left(j^{-1}\right)=\operatorname{Ln}\left(A_{2} / A_{1}\right)^{\star}\left(1 / t_{2}-t_{1}\right)
$$

$A_{1}$ et $A_{2}$ représentent la biomasse aux temps $t_{1}$ et $t_{2}$. Pour un intervalle de temps de un jour $\left(t_{2}-t_{1}\right)$ l'équation devient :

$$
\mu=\operatorname{Ln}\left(A_{2} / A_{1}\right)
$$

Le temps de génération (TG, en jour) est déterminé par la formule (REYNOLDS, 1984) :

$$
\mathrm{TG}=\mu^{-1} \operatorname{Ln} 2
$$

La biomasse algale est estimée à partir de la densité optique des cultures (mesurée à $750 \mathrm{~nm}$ ) et par comptage cellulaire aux temps $\mathrm{t}=0$ et au bout de 24 heures d'incubation.

\subsection{Ajustement aux modèles mathématiques}

Pour chaque température, la relation liant le taux de croissance à l'intensité lumineuse est décrite par le modèle de PEETERS et EILERS (1978). Ce modèle prend en considération l'augmentation de ce taux en fonction de l'intensité 
lumineuse et rend compte de la photo-inhibition aux fortes intensités lumineuses. II permet aussi d'identifier les paramètres caractéristiques de la croissance : taux maximal de croissance ( $\mu$ max), intensité lumineuse optimale (lopt), coefficient traduisant l'influence de la photo-inhibition $(\beta)$.

$$
\mu=2 \mu \max \times(1+\beta) \times I^{\prime} /\left(l^{\prime 2}+\left.2 \beta\right|^{\prime}+1\right)
$$

Avec l'= l/lopt et $\beta=$ coefficient de courbure indicateur de l'intensité du potentiel photo-inhibiteur.

Les variations des paramètres $\mu$ max, lopt et $\beta$ en fonction de la température ont été ajustées au modèle de LEHMAN et al. (1975) :

Avec $b=$ Tsup - T opt si $T>$ Topt

$$
\mu \max _{\mathrm{T}}=\mu \max x \exp \left(-2,3\left[(\mathrm{~T}-\mathrm{Topt})^{2} / \mathrm{b}^{2}\right]\right)
$$

$$
=\mathrm{T} \text { inf }- \text { Topt si } \mathrm{T}<\mathrm{Topt}
$$

$\mu$ max : taux spécifique maximal de croissance, Topt : température optimale, Tinf et Tsup : température inférieure et supérieure pour lesquelles $\mu \max _{T}=0,1 \mu \max$.

L'adéquation aux équations précitées a été estimée par la méthode des moindres carrés.

\section{3 - RÉSULTATS}

\subsection{Croissance en autotrophie}

L'évolution du taux de croissance de $M$. pusillum, en fonction de l'intensité lumineuse, pour les différentes températures, est représentée par la figure 1. L'influence de ces deux paramètres sur le taux de croissance est ajustée au modèle de PEETERS et EILERS (1978).

L'allure des courbes (figure 1) montre une évolution en deux phases : la première avec une relation linéaire entre la croissance et l'intensité lumineuse, et ce pour les intensités lumineuses inférieures à $200 \mu \mathrm{E} \cdot \mathrm{m}^{-2} \cdot \mathrm{s}^{-1}$. Au-delà de cette valeur, après un optimum, la deuxième phase est caractérisée par une diminution des taux de croissance en fonction de l'intensité lumineuse. Pour les fortes intensités lumineuses, il y a une chute du taux de croissance plus ou moins remarquable en fonction de la température. Cette chute apparaît généralement plus importante pour les températures extrêmes $15^{\circ}$ et $40^{\circ} \mathrm{C}$. Le taux de croissance maximal varie de 0,11 à $15^{\circ} \mathrm{C}$ à $0,58 j^{-1}$ à $35^{\circ} \mathrm{C}$ correspondant respectivement à des temps de générations allant de 6,3 à 1,2 jours.

Le tableau 1 récapitule les valeurs des paramètres $\mu$ max, lopt et $\beta$ associés à la croissance de $M$. pusillum. La dépendance de la croissance et de l'énergie lumineuse est visualisée par la corrélation $\left(r^{2}=0,92\right)$, hautement significative à $1 \%$, observée entre ces deux paramètres (figure 2).

On remarque que dans la formulation du modèle de PEETERS et EILERS (1978), seule l'énergie lumineuse fournie (incidente) est prise en compte. L'influence de la température est intégrée dans les expressions de $\mu$ max, lopt et $\beta$ 

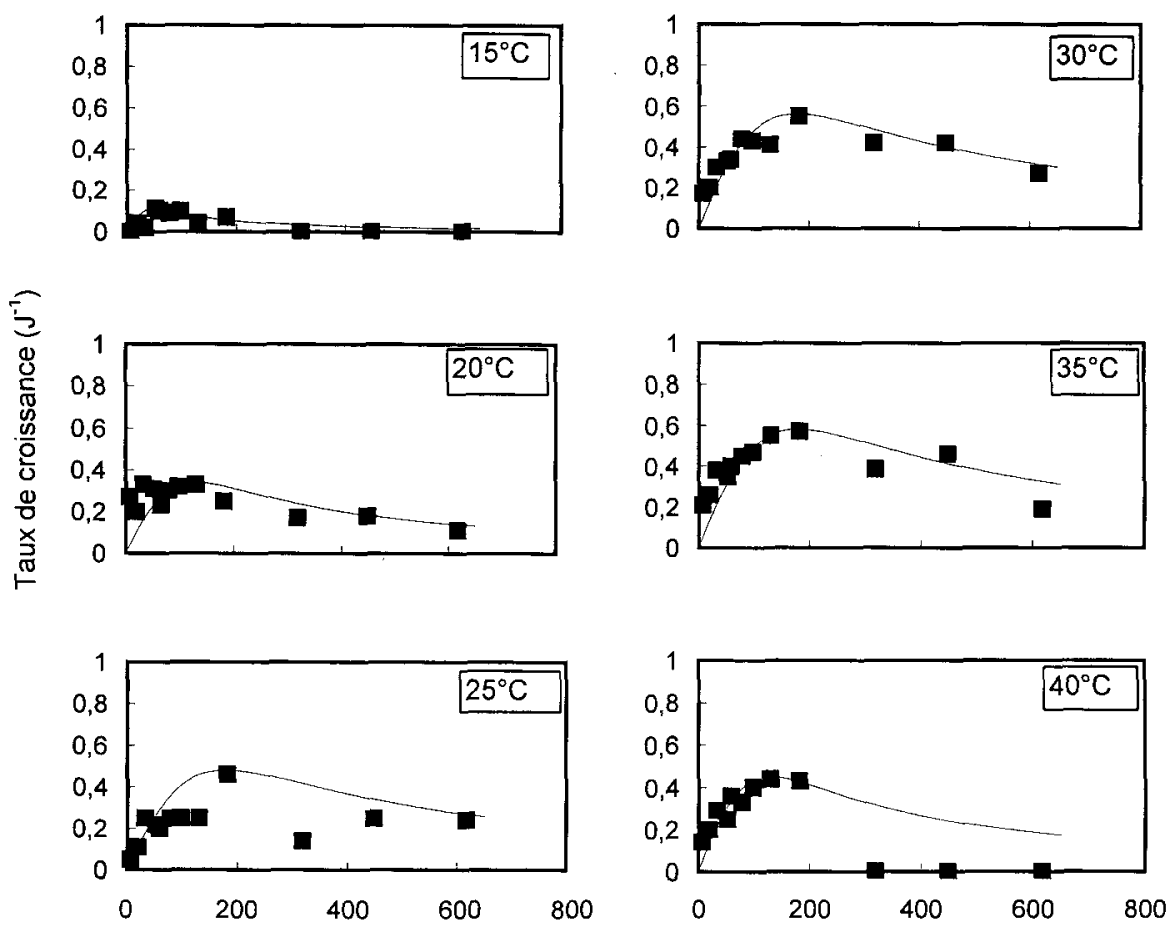

Int. lum $\left(\mu \mathrm{E} \cdot \mathrm{m}^{-2} \cdot \mathrm{s}^{-1}\right)$

Figure 1 Interaction de la lumière et de la température sur le taux de croissance de $M$. pusillum (- ajustement graphique au modèle de PEETERS et EILERS 1978).

Influence of light and temperature on the growth rate of $\mathrm{M}$. pusillum (- graphic adjusted to the model of PEETERS et EILERS 1978).

Tableau 1 Valeurs des paramètres de croissance de $M$. pusillum en autotrophie (ajustement au modèle de PEETERS et EILERS, 1978).

Table 1 Parameters of autotrophic growth of M. pusillum (adjustment to the model of PEETERS et EILERS, 1978).

\begin{tabular}{|l|c|c|c|c|c|c|}
\hline Temp $\left({ }^{\circ} \mathbf{C}\right)$ & $\mathbf{1 5}$ & $\mathbf{2 0}$ & $\mathbf{2 5}$ & $\mathbf{3 0}$ & $\mathbf{3 5}$ & $\mathbf{4 0}$ \\
\hline$\mu \max \left(\mathrm{j}^{-1}\right)$ & 0,11 & 0,34 & 0,48 & 0,56 & 0,58 &, 45 \\
$\operatorname{lopt}\left(\mu \mathrm{E} \cdot \mathrm{m}^{-2} \cdot \mathrm{s}^{-1}\right)$ & 52 & 130 & 182 & 182 & 182 & 130 \\
$\beta$ & 0,01 & 0,01 & 0,1 & 0,1 & 0,1 & 0,01 \\
\hline
\end{tabular}

(figure 3), qui suivent une loi de dépendance en fonction de la température (modèle de LEHMAN et al., 1975). Les valeurs des paramètres de $\mu$ max $=f\left(T^{\circ} \mathrm{C}\right)$, lopt $=f\left(T^{\circ} \mathrm{C}\right)$ et $\beta=f\left(T^{\circ} \mathrm{C}\right)$ associés au modèle de LEHMAN et al. (1975) sont présentées dans le tableau 2. Ces trois paramètres varient en fonction de la température suivant une fonction de type « courbe de Gauss » (figure 3). 


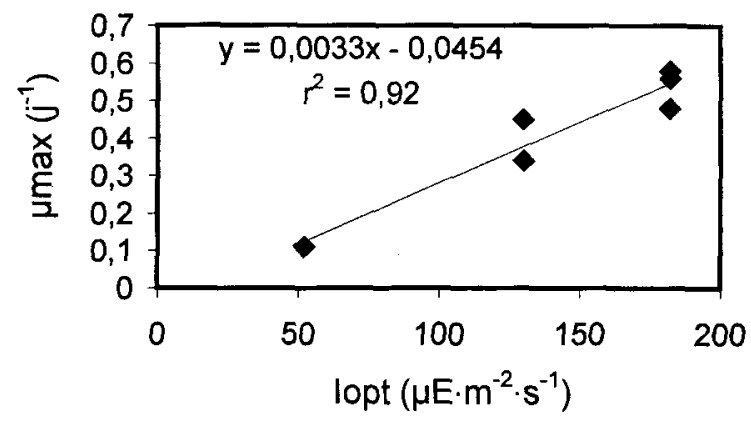

Figure 2 Corrélation entre le taux de croissance maximal ( $\mu$ max) et l'intensité lumineuse optimale (lopt) chez M. pusillum.

Correlation between maximum growth rate of M. pusillum ( $\mu$ max) and optimal light intensity (lopt).
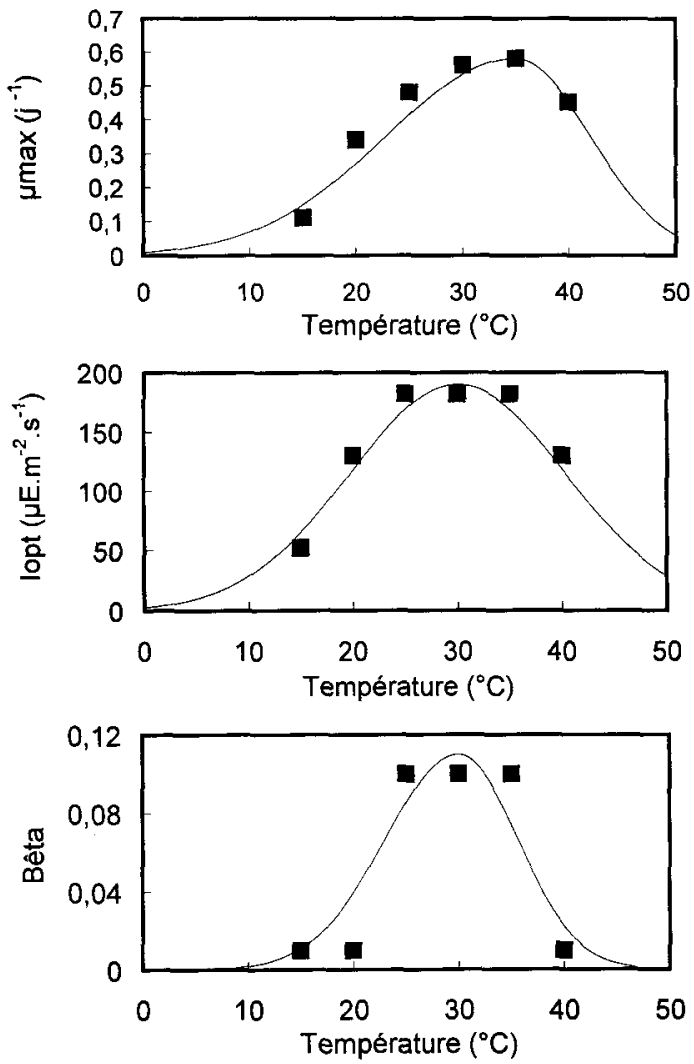

Figure 3 Variation du taux de croissance maximal ( $\mu$ max), de l'intensité lumineuse optimale (lopt) et de $\beta$ en fonction de la température ( - ajustement au modèle de LEHMAN et al., 1975).

Variation of maximal growth rate ( $\mu$ max), optimal light intensity (lopt) and $\beta$ in relation to temperature ( - graph adjusted to the model of LEHMAN et al., 1975). 
Tableau 2 Valeurs des paramètres $\mu$ max $=f(T)$, lopt $=f(T)$ et $\beta=f(T)$ (ajustement au modèle de LEHMAN et al., 1975).

Table 2 Values of parameters $\mu$ max $=f(T)$, lopt $=f(T)$ and $\beta=f(T)$ (adjustment to the model of LEHMAN et al., 1975).

\begin{tabular}{|l|c|c|c|}
\hline \multicolumn{4}{|c|}{ Température $\left(^{\circ} \mathbf{C}\right)$} \\
\hline & Tinf & Topt & Tsup \\
\hline$\mu \max =f(T)$ & 9 & 35 & 50 \\
lopt $=f(T)$ & 8 & 30 & 50 \\
$\beta$ & 15 & 30 & 42 \\
\hline
\end{tabular}

\section{$20^{\circ} \mathrm{C}$}
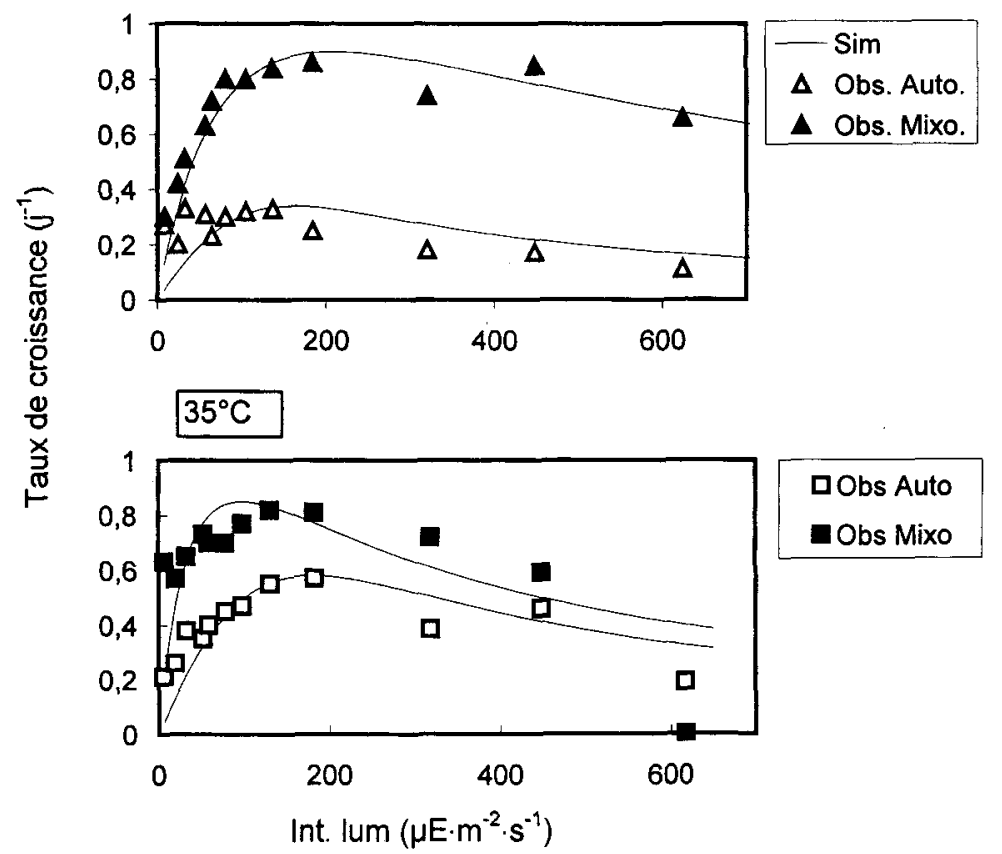

Figure 4 Effet de l'intensité lumineuse sur la croissance de $M$. pusillum en mixotrophie (mixo.) et en autotrophie (auto) aux températures $20^{\circ}$ et $35^{\circ} \mathrm{C}$ (Obs = valeurs observées ; - Sim = simulation par le modèle de PEETERS et EILERS, 1978).

Effect of light intensity on mixotrophic (mixo.) and autotrophic (auto.) growth of $\mathrm{M}$. pusillum in temperatures $20^{\circ}$ and $35^{\circ} \mathrm{C}$ (Obs = observed values; - Sim = graph adjusted to the model of PEETERS et EILERS, 1978).

\subsection{Croissance en mixotrophie}

La figure 4 montre l'effet de l'intensité lumineuse sur la croissance de $M$. pusillum en présence d'acétate aux températures $20^{\circ}$ et $35^{\circ} \mathrm{C}$. La présence d'acétate 
a conduit à une augmentation des taux de croissance. L'ajustement des valeurs expérimentales au modèle de PEETERS et EILERS a permis la détermination des paramètres de croissance ainsi que de $\beta$ en condition de mixotrophie. La comparaison des paramètres $\mu$ max, et lopt déduits de cette figure et ceux déduits de la figure 1 aux températures $20^{\circ}$ et $35^{\circ} \mathrm{C}$ (tableau 3), révèle que $\mu$ max et lopt passent, respectivement, de 0,34 à $0,93 \mathrm{j}^{-1}$ et de 182 à $222 \mu \mathrm{E} \cdot \mathrm{m}^{-2} \cdot \mathrm{s}^{-1}$ à $20^{\circ} \mathrm{C}$ d'une part, et de 0,58 à $0,85 \mathrm{j}^{-1}$ et de 182 à $150 \mu \mathrm{E} \cdot \mathrm{m}^{-2} \cdot \mathrm{s}^{-1}$ à $35^{\circ} \mathrm{C}$, d'autre part.

Tableau 3 Valeurs des paramètres de croissance de $M$. pusillum en autotrophie (auto.) et en mixotrophie (mixo.) en présence d'acétate (ajustement au modèle de PEETERS et EILERS, 1978).

Table 3 Parameters of autotrophic (auto.) and mixotrophic (mixo.) growth of M. pusillum (adjustment to the model of PEETERS et EILERS, 1978).

\begin{tabular}{|l|c|c|c|c|}
\hline Temp $\left({ }^{\circ} \mathrm{C}\right)$ & \multicolumn{2}{|c|}{20} & \multicolumn{2}{c|}{35} \\
\hline Régime trophique & Auto. & Mixo. & Auto. & Mixo. \\
$\mu \max \left(\mathrm{j}^{-1}\right)$ & 0,34 & 0,93 & 0,58 & 0,85 \\
$\operatorname{lopt}\left(\mu \mathrm{E} \cdot \mathrm{m}^{-2} \cdot \mathrm{s}^{-1}\right)$ & 130 & 222 & 182 & 150 \\
$\beta$ & 0,01 & 1 & 0,1 & 1 \\
\hline
\end{tabular}

La confrontation des courbes correspondant à l'évolution du taux de croissance en fonction de la lumière en condition d'autotrophie (figure 1) et de mixotrophie (figure 4) aux températures $20^{\circ}$ et $35^{\circ} \mathrm{C}$ a montré qu'en mixotrophie, la lumière joue, à $20^{\circ} \mathrm{C}$, aussi bien par sa présence, traduite par les très faibles intensités lumineuses, que par son intensité, alors qu'elle joue beaucoup plus par sa présence que par son intensité à $35{ }^{\circ} \mathrm{C}$. Aux faibles intensités lumineuses $\left(20 \mu \mathrm{E} \cdot \mathrm{m}^{-2} \cdot \mathrm{s}^{-1}\right)$, le taux de croissance, relevé en mixotrophie, est passé pratiquement du simple ou double $\left(0,42^{\circ}\right.$ à $20^{\circ} \mathrm{C}$ et $0,57^{\circ}$ à $\left.35^{\circ} \mathrm{C}\right)$ par rapport à celui mesuré en autotrophie $\left(0,27^{\circ}\right.$ à $20^{\circ} \mathrm{C}$ et $0,20^{\circ}$ à $\left.35^{\circ} \mathrm{C}\right)$.

\section{4 - DISCUSSION}

L'évolution du taux de croissance de $M$. pusillum en fonction de l'intensité lumineuse et de la température est ajustée au modèle de PEETERS et EILERS (1978). Pour chaque température, le taux de croissance augmente avec l'élévation de l'intensité lumineuse jusqu'à une valeur maximale ( $\mu$ max) correspondant à une intensité lumineuse optimale : lopt. Ensuite, le taux décroît sous l'effet de la photo-inhibition, qui apparaît être un mécanisme purement photochimique associé à l'intensité lumineuse élevée avec effet synergique de la température (DAUTA et al., 1990).

Le taux de croissance maximal, en autotrophie, relevé chez $M$. pusillum est faible comparativement aux taux cités chez les Chlorophycées (SCHNOOR et DI TORO, 1980). Ces auteurs soulignent que les Chlorophycées montrent, par rap- 
port aux autres familles de microalgues, les plus forts taux de croissance (entre 1 et $\left.1,75 \mathrm{j}^{-1}\right)$. Des taux supérieurs à $2 \mathrm{j}^{-1}$ sont même observés chez certaines Chlorophycées (BELKOURA et DAUTA, 1992). Le taux de croissance maximal enregistré pour $M$. pusillum se rapproche plutôt des taux trouvés chez les Diatomées (de 0,25 à $0,75 \mathrm{j}^{-1}$ ) (SCHNOOR et DI TORO, 1980). Des taux compris entre 0,4 et $1 j^{-1}$ sont généralement cités chez les Cyanobactéries (DAUTA, 1982 ; ZOHARY, 1985 ; BOUARAB et al., 1999).

La croissance de $M$. pusillum est dépendante de la température avec un Q10 de 4,36 entre $15^{\circ}$ et $25^{\circ} \mathrm{C}$. SOEDER et STENGEL (1974) ont mis en évidence l'importance de cette variable dans la croissance des microalgues et ont observé un Q10 de 6, entre $20^{\circ}$ et $30^{\circ} \mathrm{C}$, pour une souche de Chlorella. Pour M. pusillum, la température optimale de croissance est de $35^{\circ} \mathrm{C}$. Cette valeur concorde avec les valeurs citées pour les Chlorophycées et qui sont supérieures à $30^{\circ} \mathrm{C}$ (BOUMNICH et al., 1990 ; BELKOURA, 1994).

Le paramètre lopt est aussi une fonction dépendant de la température. Le Q10 est de 3,5 entre $15^{\circ}$ et $25^{\circ} \mathrm{C}$. La variation de $\mu$ max et de lopt en fonction de la température, qui est en accord avec les travaux de POST et al. (1985), REYNOLDS (1989) et OJALA (1993), met en évidence l'interdépendance entre le taux de croissance, l'intensité lumineuse et la température. Cette interdépendance avec la température constatée également pour le coefficient $\beta$ est illustrée par l'ajustement au modèle de LEHMAN et al. (1975). La température a une influence directe sur le métabolisme et par conséquent sur le taux de croissance du phytoplancton (GOLDMAN, 1977). Les fluctuations de ce facteur constituent une des limitations majeures de la production de biomasse des microalgues (TORZILLO et al., 1991). Selon VONSHAK et RICHMOND (1988) le rendement maximal de biomasse ne peut être atteint dans les bassins ouverts, utilisés pour produire la biomasse algale, à cause principalement des difficultés à maintenir la température optimale pendant toute la journée et durant toute l'année. Dans une étude comparative sur le rendement de production atteint par Spirulina dans les bassins ouverts et dans les photobioréacteurs, TORZILLO et al. (1986) ont montré que le rendement était meilleur dans ce dernier à cause du maintien de la température optimale à l'intérieur du photobioréacteur. La température influence fortement la composition chimique des cellules. À taux de croissance fixe, les vitesses d'assimilation pour le carbone et l'azote varient beaucoup avec la température (GOLDMAN, 1977). WILLIAMS (1971) a montré que l'optimum de température pour la division et la production de biomasse n'est pas nécessairement le même. Les travaux de GOLDMAN (1977) sur Dunaliella tertiolecta et Phaeodactylum tricornutum corroborent les observations de WILLIAMS (1971).

L'intensité lumineuse optimale, relevée pour $M$. pusillum, est de $182 \mu \mathrm{E} \cdot \mathrm{m}^{-2} \cdot \mathrm{s}^{-1}$. Cette valeur est inférieure, par exemple, à celle enregistrée pour Chlorella sorokiniana espèce appartenant à la même famille et isolée dans le lagunage pilote de Marrakech (BELKOURA et DAUTA, 1992). D'une manière générale, les Chlorophycées se développent à des intensités lumineuses plus élevées que celles nécessaires aux développements des Diatomées et des Cyanobactéries (BOUMNICH et al., 1990). JORGENSEN (1969) et RAPS et al. (1983) attribuent cette variation de lopt suivant les classes d'algues à des adaptations différentielles en fonction de la nature de leur composition pigmentaire.

Les effets de la température et de la lumière sur le taux de croissance des microalgues varient d'une espèce à l'autre à cause de leur stratégie adaptative 
aux changements des conditions environnementales (TALBOT et al., 1991). En fonction de l'élévation de la température, umax augmente en concomitance avec lopt. Ceci met donc en exergue l'interdépendance de la température et de l'énergie lumineuse reçue par les cellules algales. RICHMOND (1992) rapporte que l'énergie lumineuse est d'autant plus utilisée que la température est optimale. Ce fait a pour conséquence qu'une bonne partie de l'énergie lumineuse incidente en surface des écosystèmes aquatiques n'est pas utilisée de façon optimale à des faibles températures.

En plus du contrôle des processus physiologiques et métaboliques par la lumière et la température, la matière organique est indispensable pour certaines espèces de microalgues. L'utilisation du carbone organique par les microalgues a été rapportée par plusieurs auteurs (BERMAN et al., 1977 ; HELLEBUST et LE GRESDLEY, 1985 ; PEARSON et al., 1987 ; FEUILLADE et FEUILLADE, 1989 ; POST et al., 1994). L'acétate absorbé, intègre le métabolisme cellulaire par la voie du cycle de glyoxylate et par la voie du cycle tricarboxylique. Chez les végétaux et les micro-organismes, le shunt glyoxylique, considéré comme une variante du cycle de Krebs, pourvoie ce dernier, notamment en acide oxaloacétique nécessaire à sa rotation normale (NEILSON et LEWIN, 1974 ; LEHNINGER, 1981). L'utilisation de ce substrat apparaît avoir une influence sur les paramètres caractéristiques de croissance ( $\mu \mathrm{max}$, et lopt) et du coefficient $\beta$ de cette microalgue. La comparaison des paramètres $\mu$ max et lopt calculés en autotrophie et ceux calculés en mixotrophie révèle que ces paramètres ont subi une augmentation importante en mixotrophie. Le coefficient $\beta$ a connu lui aussi une élévation et passe de 0,01-0,1, en autotrophie, à 1, en mixotrophie, traduisant une certaine sténophotie de la microalgue en autotrophie et une tendance à l'euryphotie en mixotrophie. Ces résultats indiquent que les paramètres de croissance $\mu \max$, lopt et $\beta$ varient avec le mode trophique des microalgues.

La confrontation des courbes correspondant à l'évolution, en fonction de la lumière, du taux de croissance en autotrophie et en mixotrophie montre qu'en condition de mixotrophie, la lumière joue aussi bien par sa présence que par son intensité à $20^{\circ} \mathrm{C}$, alors qu'elle joue beaucoup plus par sa présence que par son intensité à $35{ }^{\circ} \mathrm{C}$. À des faibles intensités lumineuses $\left(20 \mu \mathrm{E} \cdot \mathrm{m}^{-2} \cdot \mathrm{s}^{-1}\right)$, le taux de croissance, relevé en mixotrophie, est passé presque du simple ou double par rapport à celui mesuré en autotrophie. Ce résultat est comparable à celui rapporté par SEVRIN-REYSSAC et al. (1995) qui ont montré que la Chlorophycée Scenedesmus falcatus présentait un taux de croissance accru et maximal en mixotrophie à une intensité lumineuse plus basse que celle mesurée en autotrophie.

\section{5 - CONCLUSION}

La croissance de la microalgue Micractinium pusillum en fonction de la lumière et de la température est étudiée en conditions optimales (absence de l'effet de l'auto-ombrage et d'une carence en éléments nutritionnels azote et phosphore). Les taux de croissance mesurés en fonction des variables lumière 
et température, en autotrophie et en mixotrophie, sont ajustés au modèle de PEETERS et EILERS (1978). Ce modèle a permis la définition des paramètres caractéristiques de croissance ( $\mu$ max, lopt) et du coefficient $\beta$ de la microalgue. En autotrophie à la température maximale de croissance $35^{\circ} \mathrm{C}$, ces paramètres sont : $\mu \max \left(0,58 \mathrm{j}^{-1}\right)$, lopt $\left(182 \mu \mathrm{E} \cdot \mathrm{m}^{-2} \cdot \mathrm{s}^{-1}\right)$ et $\beta(0,1)$. La dépendance de ces paramètres vis-à-vis de la température est traduite par l'ajustement au modèle de LEHMAN et al. (1975). La croissance en mixotrophie en présence d'acétate a révélé la variation de ces paramètres en fonction du régime trophique de l'espèce algale. En effet, en mixotrophie et à la même température, ces paramètres sont respectivement de $0,85 \mathrm{j}^{-1}, 150 \mu \mathrm{E} \cdot \mathrm{m}^{-2} \cdot \mathrm{s}^{-1}$ et 1 . L'utilisation de cette source de carbone a montré également le changement de la température maximale de croissance de la microalgue qui se situe probablement entre $20^{\circ}$ et $35^{\circ} \mathrm{C}$. Les paramètres $\mu$ max et lopt relevés à $20^{\circ} \mathrm{C}$ sont supérieurs à ceux mesurés à $35^{\circ} \mathrm{C}$.

La croissance en mixotrophie, comportement probable dans le lagunage naturel, a induit plus particulièrement l'élévation du taux de croissance et du coefficient $\beta$. Cette étude a montré également que l'influence de l'intensité lumineuse sur la croissance varie en fonction du mode trophique.

\section{RÉFÉRENCES BIBLIOGRAPHIQUES}

BELKOURA M., 1994. Chlorella sorokiniana Shihira \& Krauss: Écophysiologie, modélisation de cultures et perspectives de valorisation de la biomasse algale. Th. Doct. d'état Univ. Cadi Ayyad, Fac. Sci. Semlalia, Marrakech, 154 p.

BELKOURA M., DAUTA A., 1992. Interaction lumière-température et influence de la photopériode sur le taux de croissance de Chlorella sorokiniana Shihira \& Krauss. Annls Limnol., 28 (2), 101-107.

BERMAN T., HADAS O., KAPLAN B., 1977. Uptake and respiration of organic compounds and heterotrophic growth in Pediastrum duplex (Meyer). Freshwat. Biol., 7, 495-502.

BOUARAB L., OULD EL MEHDI M., SBYYAA B., AIT MELLOUL A., OUDRA B., LOUDIKI M., HASSANI L., DAUTA A., 1999. Étude expérimentale des voies d'élimination de l'azote en présence de trois microalgues - Synechocystis, Chlorella, Micractinium : évaluation de l'assimilation algale, de la nitrification et de stripping de l'ammoniac. Annis. Limnol., 35 (1), 13-22.

BOUMNICH L., DAUTA A., DEVAUX J., ROMAGOUX J.C., 1990. Influence de la lumière et de la température sur la croissance de quatre espèces d'algues d'un lac eutrophe (Lac d'Aydat, Puy de Dôme, France). Ann/s Limnol., 26 (1), 3-10.

BRUNEL L., DAUTA A., GUERRI M., 1982. Croissance algale : validation d'un modèle a stock à l'aide de données expérimentales. Annls. Limnol., 18 (2), 91-99.

CAPERON J., MEYER J. 1972. Nitrogen limited growth of marine phytoplankton. I. Changes in population characteristics with steady-state growth rate. Deep-Sea Res., 19, 601-618.

DAUTA A., 1982. Conditions de développement du phytoplancton. Étude comparative du comportement de huit espèces en culture. 1. Détermination des paramètres de croissance en fonction de la lumière et de la température. Ann/s Limnol., 18 (3), 217-262.

DAUTA A., DEVAUX J., PIQUEMAL F., BOUMNICH L., 1990. Growth rate of four freshwater algae in relation to light and temperature. Hydrobiologia, 207, 221226.

DE LA NOÜE J., DE PAUW N., 1988. The potential of microalgal biotechnology: A 
review of production and uses of microalgae. Biotech. Adv., 6, 725-770.

DERRAZ M., DAUTA A., CAPBLANCQ J., ABASSI M., 1995. Influence de la lumière et de la température sur les taux de croissance et de photosynthèse de Scenedesmus crassus Chodat, isolé de la retenue eutrophe El Kansera (Maroc). Annls Limnol., 31 (1), 65-74.

ENDO H., SANSAWA H., NAKAJIMA K., 1977. Studies on Chlorella regularis, heterotrophic fast - growing strain II. Mixotrophic growth in relation to light intensity and acetate concentration. Plant \& Cell. Physiol., 18, 199-205.

FEUILLADE M., FEUILLADE J., 1989. Heterotrophic capabilities of blue - green alga Oscillatoria rubescens. Arch. Hydrobiol., 117 (1), 61-76.

GAO K., ARUGA Y., ASAKA K., KIYOHARA M., 1993. Influence of enhanced $\mathrm{CO}_{2}$ on growth and photosynthesis of the red algae Gracilaria sp. and G. chilensis. J. appl. Phycol., 5, 563-571.

GOLDMAN J.C., 1977. Temperature effects on phytoplankton growth in continuous culture. Limnol. Oceanogr., 22 (5), 932936.

GROBBELAAR J.U., 1994. Turbulence in mass algal cultures and the role of light/dark fluctuations. J. appl. Phycol., 3, 331-335.

GUERRI M. M., BRUNEL L., DAUTA A., 1981. Interaction de la lumière et de la température sur le taux de croissance de Scenedesmus crassus. Annals Limnol., 17 (2), 97-104.

HARRIS G.P., 1978. Photosynthesis, productivity and growth: The physiological ecology of phytoplankton. Arch. Hydrobiol. Beih. Ergebn. Limonol., 10, 1-171.

HEE-MOCK O., RHEE G.Y., 1991. A comparative study of microalgae isolated from flooded rice paddies: light -limited growth, $\mathrm{C}$ fixation, growth efficiency and relative $\mathrm{N}$ and $\mathrm{P}$ requirement. $J$. appl. Phycol., 3, 211-220.

HELLEBUST J.A., LE GRESLEY S.M.L., 1985. Growth characteristics of the marine rock pool flagellate Chlamydomonas pulsatilla Wollenweber (chlorophyta). Phycologia, 24 (2), 225-229.

HERZIG R., FALKOWSKI P.G., 1989. Nitrogen limitation in /sochrysis galbana
(Haptophyceae). I. Photosynthetic energy conversion and growth efficiencies. J. Phycol., 25, 462-471.

JONES J.G., 1977. The effect of environmental factors on estimated viable an total population of planktonic bacteria in lake and experimental enclosure. Freshwat. Biol., 7, 67-91.

JORGENSEN E.G., 1969. The adaptation of plankton algae. IV. Light adaptation in different algal species. Physiol. Plant., 22 (6), 1307-1015.

KNOWLTON M.F., JONES J.R., 1996. Experimental evidence of light and nutrient limitation of algal growth in turbid midwest reservoir. Arch. Hydrobiol., 135 (3), 321-335.

LALIBERTÉ G., DE LA NOÜE J., 1993. AutoHetero-, and Mixotrophic growth of Chlamydomonas humicola (chlorophyceae) on acetate. J. Phycol., 29, 612-620.

LEHMAN J.T., BOTKIN D.B., LIKENS G.E., 1975. The assumptions and rationales of a computer model of phytoplankton population dynamics. Limnol. Oceanogr., 20 (3), 343-364.

LEHNINGER A.L., 1981. Biochimie : Bases moléculaires de la structure et des fonctions cellulaires. $2^{\mathrm{e}}$ édition, Flammarion Médecine - Sciences, $1088 \mathrm{p}$.

NEILSON A.H., LEWIN J., 1974. Phycological reviews 1 . The uptake and utilization of organic carbon by algae: an essay in comparative biochemistry. Phycologia, 13 (3), 227-264.

OJALA A., 1993. Effects of temperature and irradiance on the growth of two freshwater photosynthetic cryptophytes. J. Phycol., 29, 278-284.

PEARSON H.W., MARA D.D., MILLS S.W., SMALLMAN D.J., 1987. Factors determining algal populations in Waste Stabilization Ponds and the influence of algae on Pond performance. Wat. Sci. Tech., 19 (12), 131-140.

PEETERS J.C., EILERS P., 1978. The relationship between light intensity and photosynthesis. A simple mathematical model. Hydrobiol. Bull., 12, 134-136.

POST A.F., COHEN I., ROMEME., 1994. Characterization of two Chlorella vulgaris (Chlorophyceae) strains isolated from wastewater oxidation ponds. J. Phycol., 30, 950-954. 
POST A.F., WITT R., MUR L.R., 1985. Interactions between temperature and light intensity on growth and photosynthesis of the cyanobacterim Oscillatoria agardhii. J. Plankton Res., 7 (4), 487-495.

RAPS S., WYMAN K., SIEGELMAN H.W., FALKOWSKI P.G., 1983. Adaptation of the Cyanobacterium Microcystis aeruginosa to light intensity. Plant Physiol., 72, 829-832.

REYNOLDS C.S., 1984. The ecology of freshwater phytoplankton. Cambridge University Press, London. Beck \& Connor, $384 \mathrm{p}$.

REYNOLDS C.S., 1989. Physical determination of phytoplankton succession. $/ n$ : SOMMER U, (Ed.), Plankton ecology: succession in plankton communities. Springer-Verlag, Berlin, 9-56.

RHEE G.Y., GOTHAM I.J., 1981a. The effect of environmental factors on phytoplankton growth: temperature and de interaction of temperature with nutrient limitation. Limnol. Oceanogr., 26 (4), 635-648.

RHEE G.Y., GOTHAM I.J., 1981b. The effect of environmental factors on phytoplankton growth: Light and the interactions of light with nitrate limitation. Limnol. Oceanogr., 26 (4), 648-659.

RICHMOND A., 1992. Open systems for the mass production of photoautotrophic microalgae outdoors: physiological principles. J. appl. Phycol. , 4, 281-286.

SAKSHAUG E., ANDERSON K., KIEFER D.A., 1989. A steady state description of growth and light adsorption in the marine planktonic diatom Skeletonema costatum. Limnol. Oceanogr., 35 (1), 198-205.

SCHNOOR J.L., DI TORO D.M., 1980. Differential phytoplankton sinking and growth rates: an eigenvalue analysis. Ecol. Modelling, 9, 233-245.

SENFT W.H., 1978. Dependence of light-saturated rates of algal photosynthesis on intracellular concentrations of phosphorus. Limnol. Oceanogr., 23 (4), 709-718.

SEVRIN-REYSSAC, DE LA NOÜE J., PROULX D., 1995. Le recyclage du lisier de porc par lagunage. Tec \& Doc Lavoisier, $118 p$.
SHAFIK H.M., HERODEK S., PRESING M., VOROS L., BALOGH K.V., 1997b. Growth of Cyclotella meneghiniana Kutz. II. Growth and cell composition under different growth rates with different limiting nutrient. Annls Limnol., 33 (3), 223-233.

SHAFIK H.M., HERODEK S., PRESING M., VOROS L., BALOGH K.V., 1997a. Growth of Cyclotella meneghiniana Kutz. I. Effects of temperature, light and rate of nutrient supply. Ann/s Limnol., 33 (3), 139-147.

SOEDER C., STENGEL E.E., 1974. Physicochemical factors affecting metabolism and growth rate. In: STEWART W.D.P. [Ed.], Botanical monographs, Algal physiology and biochemistry, Univ. of California Press, Berkeley, Los Angeles, 714-732.

TALBOT P., THEBAULT J.M., DAUTA A., DE LA NOÜE J., 1991. A comparative study and mathematical modelling of temperature, light and growth of three microalgae potentially useful for wastewater treatment. Wat. Res., 25 (4), 465-472.

TORZILLO G., PUSHPARAJ B., BOCCI F., BALLONI W., MATERASSI R., FLORENZANO G., 1986. Production of Spirulina biomass in closed photobiorectors. Biomass, 11, 75-79.

TORZILLO G., SACCHI A., MATERASSI R., RICHMOND A., 1991. Effect of temperature on yield and night biomass loss in Spirulina platensis grown outdoors in tubular photobioreactors. J. appl. Phycol., 3, 103-109.

VONSHAK A., RICHMOND A., 1988. Mass production of the blue-green algae Spirulina: an overview. Biomass, 15, 233-247.

WILLIAMS F.M., 1971. Dynamics of microbial populations. In: PATTEN B.C. (Ed.), Systems analysis and simulations in ecology, v. 1, 197-267.

WYNEE D., RHEE G.Y., 1986. Effects of light intensity and quality on the relative $N$ and $P$ requirement (the optimum N/P ratio) of marine planktonic algae. J. Plankton Res., 8 (1), 91-103.

ZOHARY T., 1985. Hypersecums of the Cyanobacterium Microcystis aeruginosa in hypertrophic lake (Hartbeesport Dam. South Africa). J. Plankton Res., 7 (3), 399-406. 